\title{
Applying technologies towards safe and effective medicines
}

\author{
Allen D. Roses \\ GlaxoSmithKline, Research Triangle Park, NC, USA \\ BioTechniques 39:563-564 (October 2005) \\ doi 10.2144/000112042
}

The application of genome-based pharmacogenetics to the pipeline of drug discovery and development is gaining momentum rapidly. Instead of simply using genomics and genetics to identify new drug targets, more experiments are focused on clinical phases of the pipeline. It is now possible to anticipate specific benefits to the development of medicines. The creation of a new generation of safe and effective drugs accompanied by predictive differentiation will improve the practice of medicine. Combining new research methods with new databases can trigger momentum in fields previously mired in pregenomic technologies and informatics.

It is difficult to introduce compilations of diverse articles, but an overview of overlooked opportunities may be helpful. Therefore I have chosen four viewpoints to present-not in the context of the technologies described in the following papers, but my personal view of where some of these technologies are going and how fast these advances will come.

It is appropriate to thank the scientists and especially the scientific managers who brought genome sequencing to the world. The competition between the private and public efforts provided a tension that accelerated the process. This great technical and informatics feat occurred many years earlier than originally planned. I point this out in this introduction because that is how science actually works, many small advances that build exponentially on each other. From genome to HapMap creates more granular information that can be incorporated over time within appropriate clinical experiments to generate a body of knowledge to improve medical science.

The first vignette refers to transcriptomics, which is now an important part of tissue characterization. The biggest boost to this field, in my opinion, has been the development of standardized platforms that can be compared accurately from one experiment to another. It is hard not to be impressed by "heat maps," or redgreen-ograms of tumors. It has been more difficult to determine the functional significance of the red forests from the green forests. However, science is really about specificity of clearly relevant trees. The noise-to-signal ratios in transcriptomic experiments are very high due to coordinated expression of blocks of nearby transcripts (1). If one is looking for specificity, it is hard to decipher the lists of green and red genes by differences in expression.

Just when frustration levels are at their peak, along comes a relatively new form of expression such as short-interfering RNAs (siRNAs), which is accelerating the science of validation, replacing hordes of transgenic, knockout, or knock-in mice, and all their feeding and upkeep. The latest trend is to use these siRNAs for treatment schemes, but the critical problem of gene therapy still hangs over the enterprise-how do we introduce the siRNA to the appropriate places in the cells at the appropriate times? And then, before we know it, along comes microRNAs (miRNA), allowing another view of variable expression. The miRNA genomic map allows much greater specificity. One only needs to read the recent Lu et al. paper (2) to be impressed by the ability to profile miRNA tumor tissue expression.

A simple technical factor that should be emphasized is the advantage of moving from a technical fixed platform of comparative transcriptomics to a more flexible bead-based technique, using the fixed map of miRNAs along the genome as the reference. This is similar to single nucleotide polymorphism (SNP) profiling along the genome, with variations in mapped miRNA complimenting the SNP mapping of regions of extended disequilibrium. This distinction may be under the radar screen for many scientists, but clearly illustrates how to use new technology with new information. The genes regulated by miRNAs can also be identified and mapped. Bioinformatic methods may then be used to decipher specificity of affected metabolic pathways.

Vignette 2 involves the comparison of "whole-genome" SNP profiling applied to pharmacogenetics with the rather slow rate of identification of new susceptibility genes for complex diseases. The use of genomic-scale SNP comparisons for the association of complex gene variants requires hundreds (or thousands) of wellcharacterized patients and well-characterized controls (including ethnic variations in allele frequencies). Recognition of differences in SNP profiles that segregate in efficacy experiments or as a profile for patients at a greater risk for an adverse event is based on smaller numbers. For example, the use of tagging SNPs has also accelerated the analyses of several specific and important pharmacogenetic systems, such as the drug metabolizing enzymes. Methods that transfer the specificity of these drug metabolism variants into simultaneous screening of many variants in many people rapidly enhance their value. A single platform such as a 384-well plate with tagging SNP assay for over 52 metabolizing enzymes, including ethnic variations, is proving to have very valuable applications to real-time early (phase I and IIA side effects) drug development (3). Diagnostic companies are moving rapidly into this space, but so are academic laboratories with the ability to create custom SNP platforms on a 384-well plate. This is considerably faster and less expensive than measuring one enzyme at a time and will accelerate the application of already known science by provision of standardized platforms. This transition has occurred more rapidly than the arduous history of identifying and confirming susceptibility genes for complex diseases.

The use of appropriate platforms is also the key to wholegenome screening. It is hard to believe that we scientists "missed" the total number of human genes by a factor of three, but still made inferences about how many polymorphisms it would take to create useful SNP profiles. The difference in measuring and deciphering 60,000 versus 1.4 million SNPs is expensive. However, experiments performed with real clinical data: for susceptibility gene identification, for drug efficacy, and for drug adverse events are all currently possible-but very expensive. Platforms have been generated using interim HapMap data, which allows 500,000+ SNPs to be analyzed and interpreted simultaneously, accurately, and directly entered into analysis programs. Once the ethnic-specific population data for allele frequencies are also available in the public domain from a reasonably priced platform, academic investigators can progress rapidly with disease-specific relevant data from many relatively inexpensive clinical studies. Every physician will not need to be a technical expert to access relatively inexpensive platforms enhanced by accessible ethnic control databases in the public domain. Collections of phenotype information that have been kept in academic corrals will soon be published with genome-wide analyses, because it will no longer be necessary for the large cowboys of industry or the large academic genomic laboratories to be interested in order 
to generate data. Acquisition of new medical knowledge will be accelerated. With immediately accessible ethnic- and age-specific population data, which defines the haystack, there will be a flood of incredibly important needles flowing at an unbelievable rate.

Vignette 3 involves the rationale and momentum for using technologies and new data. In my industry, the commercial value of the future lies in safe and effective medicines provided at the correct dose and at the right time. The analysts and journalists who decide the value of this industry are frequently quoted asking the question "when will genomics provide the first blockbuster drug?" Genetics and genomics are simply tools and can already be applied. To affect medicines requires changing the culture to focusing on the patients rather than the technologies. The clinical information needs to be every bit as precise as the technical genomic measurements, but we have dangerously ignored parallel support of proper clinical experimental design. Obtaining complete phenotypic data and tissue (such as DNA and RNA) for genome-wide platform analyses over long periods of time is expensive-although not nearly as expensive as the sexiest large and necessary technical experiments of the past decade. We have simply neglected to anticipate the parallel clinical research support necessary for translation experiments. Diseases progress over time, and what is desperately needed are appropriate clinical populations with samples followed over time. Changes in a patient's condition can be monitored and translated into genetic and genomic predictive profiles, with both high positive and negative predictive values. But without detailed clinical information and appropriate samples, we just have technology.

Clinical research has a problem accepting that the only place to apply pharmacogenetics early and consistently is where drug development occurs, for the most part within the pharmaceutical industry. Most industry analysts have argued against diluting the blockbuster-one drug for everyone-while most patients want the drug that will be effective for them and produce no side or adverse effects. In fact, most healthcare providers will value differentiation by being able to reimburse those medicines with more predictable efficacy. So why has this not happened already?

The real question is not when will genomics produce a drug from some new target (7-12 years after identification of a successful target in a regulated process), but when will well-differentiated medicines reach the market with much of that drug-specific information already known? Think about it. We now learn about many uncommon adverse events and get some specific science applied when the academic medical establishment has a chance to follow a marketed drug in the people who take the medicine. To do this in drug development, the people who develop the drugs are the only ones who have access to consent and test the patients in the trials. In fact, examples of efficacy have been published. Figure 1 in Reference 4 shows a simple example of an informative phase IIA experiment. Even if the drug ran into no regulatory or safety problems, it still would be 3 years until the drug could be approved via multiple trials with 6-month treatment periods for each component study in phase IIB or phase III. In fact, examples of safety pharmacogenetics have also been published, but have been viewed by analysts only as risks. What about the benefit of being able to define efficacy and side effects early in development, to work out estimates of positive and negative predictive value of pharmacogenetic profiles, and to test people before they take the drug? Individual digitalized information will provide the bandwidth for looking at standardized SNP platform data in a cost-effective manner for patients. Schemes for postmarketing surveillance for the regulators and for the population in general are urgently needed so that individuals can reap the benefits of prediction and treatment.

Analyses of the pharmaceutical industry frequently focus on those blockbusters that have had to be withdrawn from the markets. It is now, in 2005, possible to envision choices of systems that make safe and effective medicines for the right patient possible. Imagine in the summer of 2005 if there were a COX2 inhibitor with defined segmented efficacy and pharmacogenetics monitored safety in mid-development. Imagine being able to provide data that says "Our drug is safer than the others, and we can help predict in whom we can expect efficacy." Imagine if we could do this with virtually all drugs. It is no less an expectation than sequencing the Human Genome was in 1990. This difference will not be an "I am finishedthe genome is complete" end point. Rather, each new developed drug with standards of efficacy and safety will erode other markets over time and will eventually include over-the-counter medications, generic drugs, and nontraditional therapies. All risky phenotypes are fair game.

Vignette 4 impacts on my <2000 word limit to summarize pharmacogenetics as an introduction. In brief, GlaxoSmithKline (GSK) has made a considerable investment (but dwarfed by the loss of billions of dollars from a single withdrawn blockbuster) over most of a decade in genome-wide pharmacogenetics and pharmacogenomics. GSK has found accurate "diagnostics" for side-effects such as diarrhea, hyperbilirubinemia, and hypersensitivity syndrome, as early as early phase I and phase IIA programs (4). With these diagnostics, the risk of success is raised for candidate molecules as they move towards GSK's published asset portfolio. GSK is monitoring development programs as a corporate policy and uses proteomics and genomics to determine the mode of action of lead molecules identified by "black box" library screens. GSK has programs in $>18$ different diseases to detect tractable targets-screening more than 15 million SNPs against $>1000$ well-phenotyped, prospectively ascertained patients and $>1000$ controls - with appropriate statistical permutations (5). These studies will be extended with the production of $500 \mathrm{~K}$ SNP chips. GSK is also conducting large studies of several important rare adverse events, such as long QT, Stevens-JohnsonSyndrome, ischemic colitis, and predictors of cardiac risks, and also new targets, genetically validated leads, and pharmacogenetic coverage of clinical development programs to increase the prediction of efficacy and to minimize risks-and it is happening today below most radar screens. The mandate of genome-wide science is really all about helping the patients by using technological tools appropriately and inexpensively. As a research enterprise, we should invest in sufficiently large clinical experimental designs (prospectively phenotyped association or epidemiology studies) so that our complicated and always improving technologies can be effectively applied at a reasonable expense in a practical time frame. Imagine the contribution to clinical outcome studies if the Framingham Study had collected DNA and other relevant tissue samples from the beginning?

\section{REFERENCES}

1.Chang, L., S. Ghosh, D.B. Searls, A.M. Saunders, J. Cossman, and A.D. Roses. Clusters of adjacent and similarly expressed genes across normal human tissues complicates comparative transcriptomic discovery. OMICS (In press).

2. Lu, J., G. Getz, E.A. Miska, E. Alvarez-Saavedra, J. Lamb, D. Peck, A. SweetCordero, B.L. Ebert, et al. 2005. MicroRNA expression profiles classify human cancers. Nature 435:834-838

3. Roses, A.D. 2004. Pharmacogenetics and drug development: the path to safer and more effective drugs. Nat. Rev. Genet. 5:645-656.

4. Roses, A.D. 2002. Genome-based pharmacogenetics and the pharmaceutical industry. Nat. Rev. Drug Discov. 1:541-549.

5. Roses, A.D., D.K. Burns, S. Chissoe, L.T. Middleton, and P. St. Jean. 2005 Disease-specific target selection: a critical first step down the right road. Drug Discov. Today 10:177-189.

Address correspondence to:

Allen D. Roses

Senior Vice President, Genetics Research

GlaxoSmithKline

Five Moore Drive, 5-5616

Research Triangle Park, NC 27709, USA

e-mail: allen.d.roses@gsk.com 